\title{
Label-free multiplex immunoassay of AFP, CEA and CA19-9 by integrated microfluidic biosensor based on imaging ellipsometry
}

\section{Chenghong Huang, QingLin Yang, Fayi Song, Nan Chen, Bo Yao, Xiaoling Liao, ShiTong Zhang, Yanyan Chen \& Gang Jin}

To cite this article: Chenghong Huang, QingLin Yang, Fayi Song, Nan Chen, Bo Yao, Xiaoling Liao, ShiTong Zhang, Yanyan Chen \& Gang Jin (2016) Label-free multiplex immunoassay of AFP, CEA and CA19-9 by integrated microfluidic biosensor based on imaging ellipsometry, Integrated Ferroelectrics, 171:1, 59-69, DOI: 10.1080/10584587.2016.1171664

To link to this article: http://dx.doi.org/10.1080/10584587.2016.1171664

\section{Published online: 27 May 2016.}

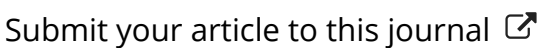

Џ Article views: 7

Q View related articles $₫$

View Crossmark data ¿ 


\title{
Label-free multiplex immunoassay of AFP, CEA and CA19-9 by integrated microfluidic biosensor based on imaging ellipsometry
}

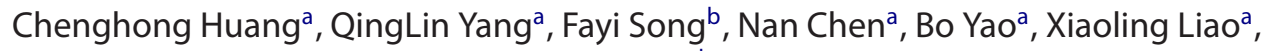 \\ ShiTong Zhang a, Yanyan Chenc, and Gang Jin ${ }^{\text {d }}$ \\ a Institute of Biomaterials and Living Cell Imaging Technology, Chongqing University of Science and \\ Technology, Chongqing,China; ${ }^{b}$ Institute of Biomaterials and Biomedical Engineering, University of \\ Toronto, Toronto, ON, Canada; ' 'Suzhou Institute of Nano-Tech and Nano-Bionics, CAS, Suzhou, China; \\ ${ }^{\mathrm{d}}$ Institute of Mechanics, CAS, Beijing, China
}

\begin{abstract}
This study describes the development of alpha-fetoprotein (AFP), carcinoembryonic antigen (CEA) and carbohydrate 19-9(CA19-9) multiplex immunoassay by integrated microfluidic device based on imaging ellipsometry (IMBIE). Different strategies for AFP, CEA and CA 19-9 simultaneous immunoassay on the same aldehydesubstrate were developed. Unifying the reaction steps of CA19-9 for antigen direct detection, CEA for secondary antibody (rabbitantiCEA) addition, AFP for secondary (rabbit antiAFP) and an enhancer (goat lgG) application, and comprehensively considering the dynamic ranges and detection sensitivities, we combined these three markers into multiplex analysis in serial format.The sensitivities and dynamic ranges for CA19-9, CEA and AFP were $10.0 \mathrm{U} / \mathrm{mL}, 1.0 \mathrm{ng} / \mathrm{mL}, 1.0 \mathrm{ng} / \mathrm{mL}$ and $2.0-100.0 \mathrm{U} / \mathrm{mL}, 0.1-64.0$ $\mathrm{ng} / \mathrm{mL}, 1.0-128.0 \mathrm{ng} / \mathrm{mL}$, respectively. The intra-assay and interassay (coefficient variations) CVs of CA19-9, CEA and AFP were between $1.6-8.9 \%$ and $2.1-7.8 \%$. The specificities tested for both cross-reaction and heterogeneous proteins of CA19-9, CEA and AFP were not more than $6.7 \%$. Quantitative detection of 49 sera from gastric patients demonstrated that the accuracy for gastric cancer diagnosis was $83.4 \%$. The total detection time is only $60 \mathrm{~min}$ for an automated process. The proposed assay has the potential for practical application.
\end{abstract}

\section{ARTICLE HISTORY}

Received 7 August 2015

Accepted 3 February 2016

\section{KEYWORDS}

Imaging ellipsometry; immunoassay; AFP; CEA; CA19-9

\section{Introduction}

The risk of cancer to people all around the world is obvious. With the rapid increase of morbidity the mortality, early diagnosis and treatment of cancer are the keys to improvement of patients' survival rate [1]. As the levels of cancer markers in serum are heavily associated with the stages of cancer, sensitive determination and accurate analysis of cancer markers are very important to early screening and 
management of cancer [2]. Thus, great efforts have been made to develop novel immunoassay such as electrochemical [3], optical [4] and mass technique [5], for the analysis of cancer markers. On the other hand, owing to the limited specificity of single marker for an individual cancer diagnosis, multiplex analysis by a panel of cancer markers to improve the diagnostic accuracy with acceptable sensitivity and specificity have attracted considerable attentions [6-7]. Therefore, development of multiplex analysis for a panel of cancer markers is of interest as it can offer much information of patients

Fortunately, precise microfluidic control and manipulation of liquids in geometrically controlled miniaturized channels by integrated system even Lab-on-a-Chip (LOC) devices offered the feasibility of multiplex analysis within a little sheet of substrate. Thus, the microfluidic platforms for multiplex analysis of cancer markers, which created by patterning of different markers in a single LOC device, have attracted more attentions due to its capacity of high throughput for biological applications in sensing. Biosensor based on imaging ellipsometry (BIE) is a typical example. Since the concept of BIE put forward in 1995 [8], the application of BIE to perform sensitive, real-time detection of protein especially biomarkers has been greatly progressed to realize integrating microfluidic channel with the BIE system(IMBIE). Its sensitivity can up to sub-nanometer level [9-10]. However, the ability of reported researches to measure proteins through surface binding with high sensitivity only deals with detection of single biomarker. The major challenge in the application of IMBIE for clinic is to achieve multiplex analysis with a panel of markers because the sensitivity, cut-off value and various reaction steps for multiple markers must be simultaneously unified [11]. The achievement of this aim requires that the assay system has advantages of high sensitivity, short time, and easy-operation by semi/fullautomation, which still scarcely reported till now.

Recently, we reported that IMBIE was able to detect CA19-9 in undiluted human serum with sensitivity of $10.0 \mathrm{U} / \mathrm{mL}$ when operated by a one-step procedure [12]. In that work, the capture antibody was immobilized on silicon substrate coated with a carboxyl-terminated substrate. In another literature [13], we introduced that using biological signal amplification method for detection of AFP by sandwich immunoassay can increase sensitivity up to $1.0 \mathrm{ng} / \mathrm{mL}$ with glutaraldehydefunctionized silicon substrate. In the present study, we develop that using different assay strategies to accomplish multiplex analysis of AFP, CA19-9 and CEA meeting the requirements of high sensitivity and short time in undiluted whole human serum by identical immobilization of these three capture antibodies with the same immobilization method. An obvious contribution of this way would be the direct detection of the amount of captured antigen without labeling any substances, using one of the available techniques for the measurement of thin protein films on solid surfaces with a fully-automated sampling system. The results by IMBIE were statistically evaluated and compared with that of the gold standard by electro-chemiluminescence immunoassay (ECLIA). The potential of IMBIE was finally discussed and viewed. 


\section{Experimental}

\subsection{Materials and reagents}

Polished silicon wafers were purchased from General Research Institute for Nonferrous Metals (China). The standard AFP was obtained from National Institution for the Control of the Pharmaceutical and Biological Products (China). Rat anti-AFP monoclonal antibody (rat-mAb) was purchased from Meridian Life Science, Inc. Rabbit anti-AFP polyclonal antibody (Rabbit-pAb), goat-IgG, Human carcinoembryonic antigen (CEA), Rat anti-human CEA monoclonal antibody (rat-mAb), rabbit anti-human CEA polyclonal antibody (rabbit-pAb) were obtained from Beijing Kangwei Co. (China). Human CA19-9 and anti-human CA19-9 monoclonal antibody (Lot C081001, Clone 1116-NS-19-9) were purchased from Shanghai LincBio Science Co. 3-aminopropyltriethoxysilane (APTES) and glutaraldehyde(GA, $50 \%$ aqueous solution) are bought from ACROS. All other reagents were of analytical grade. Water was obtained from a millipore Milli-Q ion exchange apparatus. Phosphate-buffered saline (PBS, $8 \mathrm{mM} \mathrm{Na}_{2} \mathrm{PO}_{4} \cdot 2 \mathrm{H}_{2} \mathrm{O}, 2.68 \mathrm{mM} \mathrm{KCl}, 1.14 \mathrm{Mm}$ $\mathrm{KH}_{2} \mathrm{PO}_{4}, 137 \mathrm{mM} \mathrm{NaCl}$; $\mathrm{pH}$ 7.4) was prepared in deionized water. PBST $(0.05 \%$ tween-20) was used as washing solution. Patient sera were taken from Radiation Medical Institute of Shandong Academy of Medical science, China.

\subsection{Integrated microfluidic BIE platform}

The experiments were carried out with IMBIE developed in our laboratory. Imaging ellipsometry was an enhancement of standard single-beam ellipsometry, which combined the power of ellipsometry with microscopy and worked in the off-null mode. The imaging ellipsometry used in this study was an automated one, and ellipsometric conditions could be controlled by autoadjusting the polarizer and the analyzer; the angle of incidence was variable from 45 to $90^{\circ}$ with a resolution of $0.05^{\circ}$; the magnification of image to object is modulated according to the dimension of the field of view, so that the lateral resolution of $3 \mathrm{~mm}$ could be reached; autofocusing was realized with the standard of the Laplacian algorithm. All the adjustments were carried out automatically with microstepping motors controlled by a computer with homemade software. With the same software, the digital images in grayscale format (8 bits, 0-255 Gycale) could be also automatically captured and processed. The light source was a xenon lamp, and a specific collimating system was used to provide an expanded parallel probe beam with a diameter of $25 \mathrm{~mm}$. The beam passed through a polarizer and a compensator (a quarter wave plate) and finally onto the sample at an incident angle of $75^{\circ}$. An optical filter at $633-\mathrm{nm}$ wavelength was placed in the incident optical passage to select a wavelength in order to increase the ellipsometric contrast of image. The reflection beam passed through an analyzer and an imaging lens with a spatial filter located at its focus plane, and then the ellipsometric image was focused onto the sensing area of the CCD camera. For a sample with lateral distribution of layer thickness (or surface concentration), null ellipsometry could not 
be carried out over the entire surface simultaneously due to the fact that different areas would yield different polarization changes. To overcome the problem, the optical components in the immunosensor were adjusted to fulfill the null conditions on a silicon wafer without adsorbed layers, and the off-null ellipsometric principle was used to measure the adsorption layer thickness (or surface concentration). Under this condition, the detected intensity " $I$ " was related to the thickness $(d)$ of the layer according to $I=k d^{2}$. As for the same protein and the same ellipsometric conditions, $k$ is a constant and can be determined by the protein layer with known intensity in grayscale and its absolute thickness.

The reactor consists of a sample delivering device and a microreactor for manufacturing protein microarray. The sample delivering device includes a 24 microfluidic channel dispensing pump that can convey sample solutions by multiple parallel channels using the Teflon tubes connection under the conditions that the negative pressure was generated. When silicon substrate was attached on the PDMS template, in which embedded with $4 \times 6$ slot, 24 reaction microcells were airtightly formed. Therefore, biomolecule can couple with the substrate through microfluidic patterning to form rectangle dots in array format. The physic size for each dot is $1.0 \times 1.5 \mathrm{~mm}$. As different channels are independent and can be individually controlled, different dots between laminar flow streams containing different concentrations of biomolecules were performed to obtain experimental conditions. This indicates that different molecules will patterned within a small area on substrate to implement single marker detection in serial format or multiple marker detection in parallel format. In this style, the advantages of this fashion are (i)creating a multiplex dots on a single substrate for high throughput analysis; (ii)utilizing chemistry modified microfluidic patterning substrate to introduce functional groups onto the substrate surface to covalently immobilize the biomolecule, thus overcoming the problems posed by the physical immobilization of biomolecules by loss of biological activities; (iii) drastically reducing the detection time by integrating multiple procedures into a successive process; (iv) resulting in a microfluidic device capable of withstanding high flow rates through irreversible binding; (v) controlling the patterning outcome by flow conditions and concentration of the biomolecules in each flow of channel; (vi) packaging the biomolecules in airtight environment in favor of keeping its biological activities.

\subsection{Array design}

The lay out of the microarray was illustrated in Fig. 2. Total 24 dots were arranged in $4 \times 6$ format. A line was used as control containing a blank control and a positive control for each marker. Detailed assignments as follows: dot a1 is served as blank control that antiCA19-9 was only immobilized and a2 as positive control that 37.0 $\mathrm{U} / \mathrm{mL}$ CA19-9 was added after the immobilization of antiCA19-9. There is a little difference for CEA, that is to say, dot a3 is used as blank control that antiCEA was immobilized in addition to the application of rabbit antiCEA. But for a4 is addition of $4.0 \mathrm{ng} / \mathrm{mL}$ of CEA except for the immobilization of rat antiCEA and addition of 


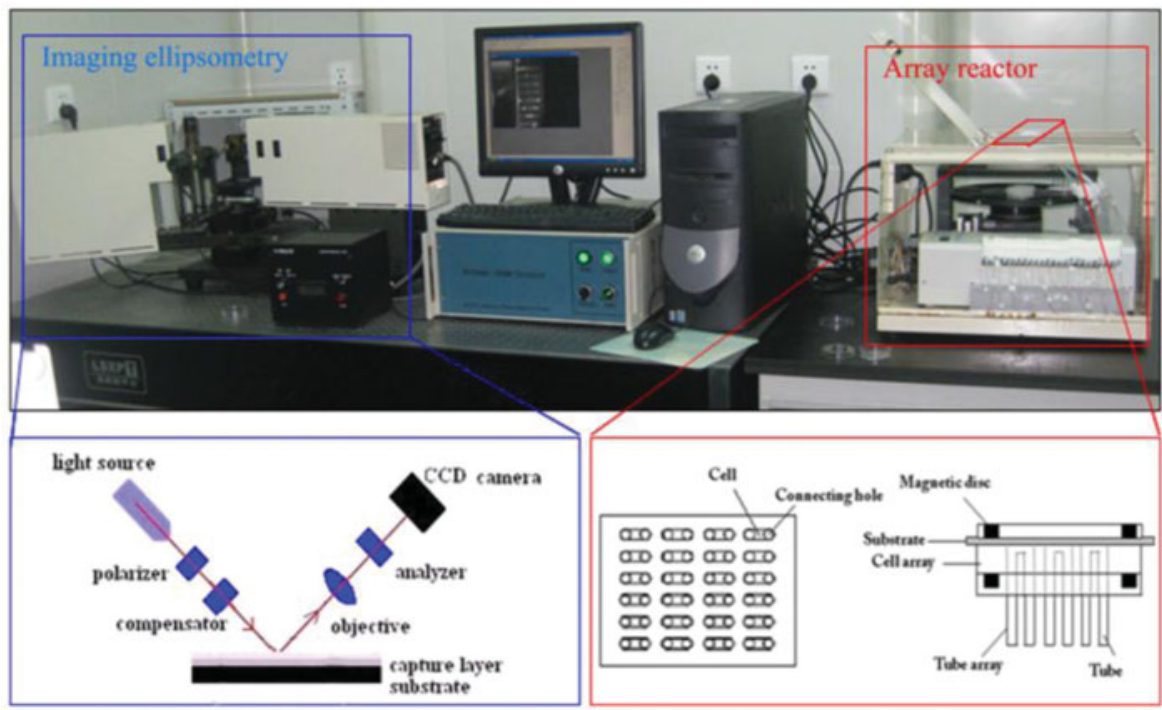

Figure 1. Illustration of IMBIE platform. It consists of imaging ellipsometry and a reactor in which embedded microreactor for array fabrication and a sample delivering apparatus for biomecules transferring driven by a 24 microfluidic channel pump. The enlarged inset shows a $4 \times 6$ microcell on PDMS template and each one contains a inlet and a outlet resulting in the formation of a square dot on substrate in microarray format by microfludic patterning.

rabbit antiCEA after CEA addition. The status for AFP is quite different from the former two. In other words, dot a 5 become blank control that rat antiAFP was only immobilized besides the application of rabbit antiAFP and goat IgG. But for dot a6, application of $25.0 \mathrm{ng} / \mathrm{mL}$ of AFP was further applied in addition of rabbit antiAFP and goat IgG for amplification of biological signal. By this way, different detection strategies were performed for these three markers to achieve different sensitivities and detection dynamic ranges. The rest dots in each row were used for clinic sera. It must be pointed out that this can be executed by different reaction steps and unified by independent microfluidic channels.

\subsection{Configuration of immunoassay}

An immunoassay is a specific type of biochemical test that measures the presence or concentration of a substance in solutions (serum or urine) that frequently contain a complex mixture of substances. For antigen analysis, the most adopted, currently, configuration is sandwich form. Namely, the antigen is bound to the antibody site (primary antibody), then another labeled antibody (secondary antibody) is bound to the antigen so that the amount of the labeled antibody on the different site will be directly proportional to the concentration of the antigen. Labeling of antibody often result in loss of its activity, thus label-free detection is usually welcomed because the native antibody can preserve its inherent molecular structures. In this paper, the configuration for CA19-9, CEA and AFP was separately designed taking into account their reaction time, dynamic ranges and detection sensitivities. For CA19-9, the configuration of direct detection was taken 


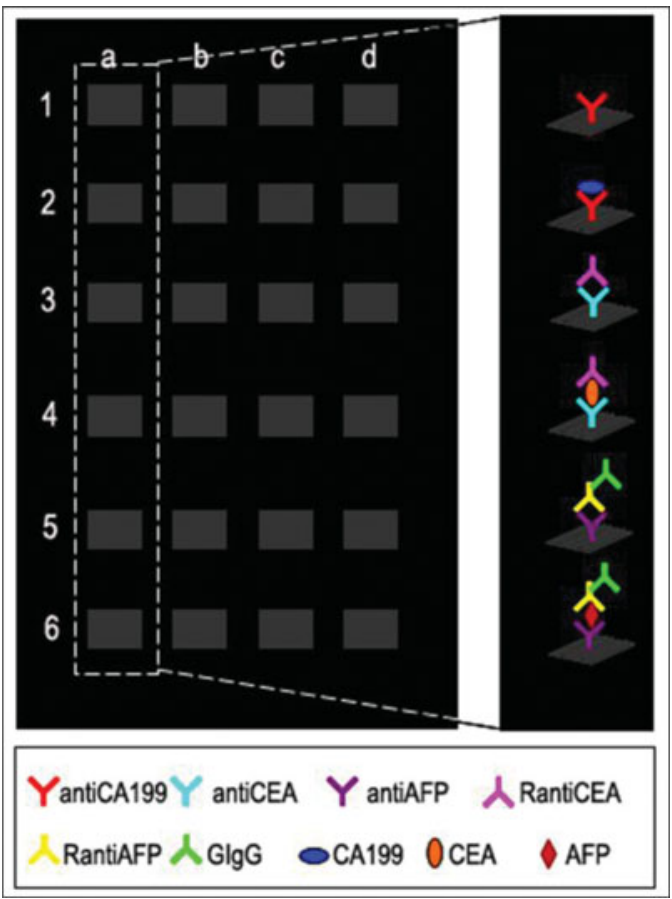

Figure 2. Lay out and Configuration of immunoassay in the microarray. The a line was served as reference, in which a1 is for blank control by only immobilization of antiCA19-9 and a2 is for positive control by $37.0 \mathrm{U} / \mathrm{mL}$ CA19-9 addition after antiCA19-9 immobilization. Similarly, a3 is for blank control by only immobilization of antiCEA and a 4 is positive control by addition of $4.0 \mathrm{ng} / \mathrm{mL}$ CEA after immobilization of antiCEA. a5 is for blank control by only immobilization of antiAFP and a6 is positive control by addition of $25.0 \mathrm{ng} / \mathrm{mL}$ AFP after antiAFP immobilization as well as addition of rabbit antiAFP and goat IgG. The others dots in each row are used for corresponding sera application.

through antigen capturing by immobilized primary antibody. But for CEA, secondary antibody (polyclonal antibody) was added after the antigen captured by immobilized primary antibody. For AFP, there is a little difference that an enhancer was added by addition of goat IgG to combine with the secondary antibody (polyclonal antibody) after it was bound with the captured antigen (The enlarged inset of lin1 in Fig 2.).

\subsection{Microfluidic patterning and immunassay}

The modification of silicon substrate was referred to the literature [14]. Briefly, silicon substrate was initially treated with piranha solution $\left(30 \% \mathrm{H}_{2} \mathrm{O}_{2}: 70 \% \mathrm{H}_{2} \mathrm{SO}_{4}=\right.$ $1: 3, \mathrm{v} / \mathrm{v}$ ) for $30 \mathrm{~min}$ followed by intensively rinsing with deionized water. The oxidized silicon wafer was immersed in ethanol solution containing APTES (5\%) for $2 \mathrm{~h}$ incubation. After washed by ethanol and deionized water repeatedly, the modified silicon wafer was placed into a mixture of glutaraldehyde (GA)/PBS $(1: 10, \mathrm{v} / \mathrm{v})$ to react $1 \mathrm{~h}$, rinsed with $\mathrm{PBS}$ and deionized water again. The prepared substrate was stored at $4^{\circ} \mathrm{C}$ for later use.

Prior to microfluidic patterning, the prepared wafer was reversely impressed on the PDMS template and the airproof was checked. The immobilization of rat 
antiCA199, rat antiCEA and rat antiAFP was simultaneously carried out by in parallel format. Namely, the prepared monoclonal antibody solutions were separately transported to the reaction microcells with a flow rate of $1.0 \mu \mathrm{L} / \mathrm{min}$ for $20 \mathrm{~min}$. After washed by PBS with a flow rate of $10 \mu \mathrm{L} / \mathrm{min}$ for $5 \mathrm{~min}$, the substrate surface immobilized antibodies were blocked by $1.0 \mathrm{mg} / \mathrm{mL}$ BSA with a flow rate of $1.0 \mu \mathrm{L} / \mathrm{min}$ for $20 \mathrm{~min}$ so that achieve the microfluidic patterning process. Next, antigen including CA19-9 (37.0 U/mL), CEA $(4.0 \mathrm{ng} / \mathrm{mL})$ and AFP $(25.0 \mathrm{ng} / \mathrm{mL})$ or PBS solution was individually added to the corresponding positive control cell or blank control cell with a flow rate of $1.0 \mu \mathrm{L} / \mathrm{min}$ for $60 \mathrm{~min}$ (CA19-9), $40 \mathrm{~min}$ (CEA) and $20 \mathrm{~min}$ (AFP) reaction, respectively. Except for the differences of antigen reaction time, rabbit antiCEA was further added to react with the combined CEA with a flow rate of $1.0 \mu \mathrm{L} / \mathrm{min}$ for $15 \mathrm{~min}$ and washing by PBST for $5 \mathrm{~min}$. rabbit antiAFP and goat IgG were separately added to react with the combined AFP with a flow rate of $1.0 \mu \mathrm{L} / \mathrm{min}$ for $15 \mathrm{~min}$ and washing by PBS for another $5 \mathrm{~min}$. As such, the total reaction time for these three cancer markers detection was unified as $60 \mathrm{~min}$ and reaction steps were actually a continuous and autoprocess. All above operations were performed at ambient temperature $\left(23-27^{\circ} \mathrm{C}\right)$.

\subsection{Statistical analysis}

Parametric results of healthy and patient group were expressed as mean \pm standard deviation (SD) and difference between groups was compared by independentsamples Test. Comparison between BIE and ECLIA was made using bivariate Spearman's correlation test (A $P$ value $<0.05$ was considered as statistically significant). Agreement was performed by kappa analysis.

\section{Results and discussions}

\subsection{Microfluidic patterning of capture antibodies}

The biosensor uses a sensing surface which is prepared by predeposition of different antibodies in separate areas on silicon substrate. In this way, a test surface is obtained with the desired type of antigen, its layer thickness, and location on the surface being predetermined for each area. Such a surface is incubated with the solution containing or not corresponding antigen. The antibody molecue will then catch the antigen accompanied the layer thickness increase in the corresponding dot. A significant increase of the layer thickness indicates that the solution contains the particular antigen. By using ellipsometry for imaging the thickness distribution among the different dots, the existence of one or more particular type of antigen in the solution can thus be deduced. The intensity $I$ of layers was proportional to the square of the thickness of the layers, so the different grayscales of layers indicated different thickness of layers. The higher the intensity and the brighter of the image, the higher the thickness. The dots can also be intuitively presented in form of image and can be further expressed by $3-\mathrm{D}$ so that more details at sub-nanometer level of the 


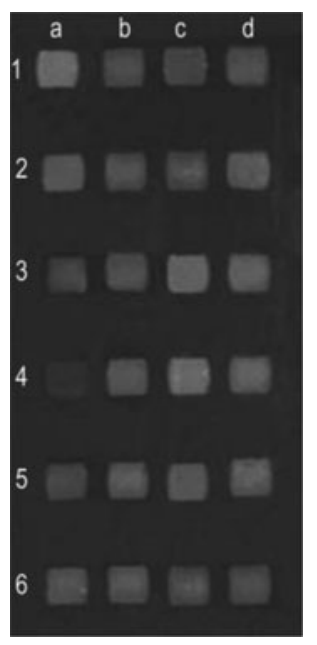

Figure 3. Typical ellipsometric image obtained by immobilizing antiCA19-9, antiCEA and antiAFP on modified silicon substrate for corresponding CA19-9, CEA and AFP detection. Detailed descriptions are indicated in the text.

image can be displayed. Fig. 3 shows typical ellipsometric image by immobilizing antiCA19-9, antiCEA and antiAFP on modified silicon substrate for corresponding CA19-9, CEA and AFP detection on the basis of the microarray design in 2.3. The grayscale of a1 is 106.56 for antiCA19-9 blank control. When $37.0 \mathrm{U} / \mathrm{mLl}$ CA 19-9 applied, the grayscale of a2 for positive control was increased to 152.12 . Then, positive or negative result profile for the rest dots in row 1 and 2 can be judged relying on their grayscales. Similar status also can be carried for antiCEA and corresponding CEA combination in row 3 and 4, and for antiAFP and AFP in row 5 and 6. In this way, total six samples containing three cancer markers information can be simultaneously determined in a single substrate.

\subsection{Assay optimization}

In this paper, within the measurable ranges for ordinary ECLIA of the tumor markers, concentration of capture antibody for antiCA19-9, antiCEA and antiAFP was firstly optimized and $100.0 \mathrm{mg} / \mathrm{mL}$ was entirely adopted. Except for CA19-9, the work concentrations of rabbit antiCEA and antiAFP as well as goat IgG were optimized. For rabbit antiCEA, solutions with diluted ratio of 1:5, 1:10, 1:15, 1:30 and 1:50 was added to combine with captured CEA to obtain optimal dose response and best detection limit. There was a significant difference between efficacy of different concentrations of rabbit-pAb and grayscale response. Ratio lower than 1:15 resulted in a narrower dynamic range and higher than 1:20 led to the dynamic range beyond the grayscale of the system, so this ratio was selected as work concentration. For rabbit antiAFP and goat IgG, test was performed in the form of orthogonal model. After capture antibody immobilized and addition of AFP, different diluted ratio of rabbit antiAFP $(1: 5,1: 10,1: 20,1: 30$ and $1: 40)$ and goat $\operatorname{IgG}(1: 5,1: 10,1: 20,1: 30$ and $1: 40$ ) was added to evaluate grayscale response. By evaluation of the dynamic range 

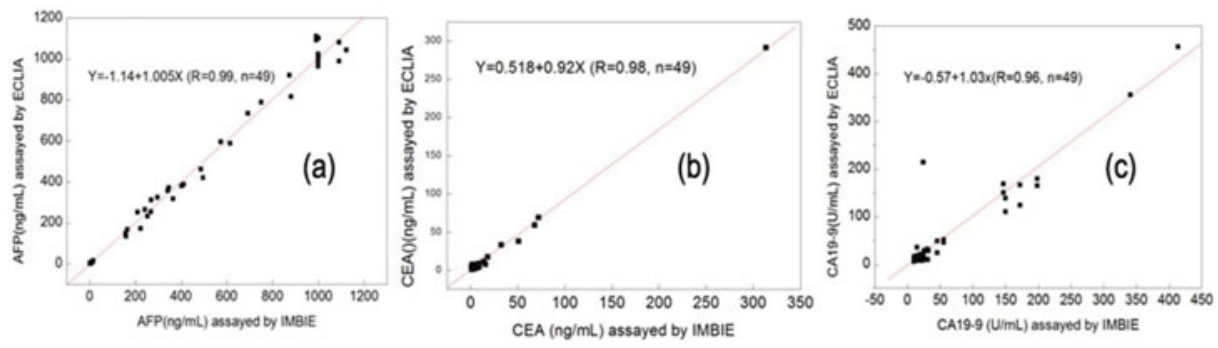

Figure 4. Correlation analysis of AFP (a), CEA (b) and CA19-9 concentrations in 49 sera from patients between IMBIE assay and conventional ECLIA assay.

and sensitivity, we selected the dilution ratio 1:20 of rabbit antiAFP and 1:30 of goat $\mathrm{IgG}$ as the work concentration.

\subsection{Detection performances for CA19-9, CEA and AFP}

Qualitative detection for 49 sera from gastric patient was first conducted. The positive rates of $64.0 \%$ for AFP (25/49), 38.6\% for CEA (18/49) and 59.2\% for CA19-9 were statistically calculated. The accuracy for gastric cancer diagnosis was $83.4 \%$ through binomial regression analysis.

Our objective was to develop the facile method to be applicable to a broad test menu as well as to provide a fully automated and rapid immunoassay strategy. To accomplish this, we needed a total immunoassay time no longer than $60 \mathrm{~min}$. Regardless of analytes, we thus chose $20 \mathrm{~min}$ for the immunorereaction, attainable with the use of fluidic channel, and performed the following evaluations by means of the immunoassay formats descried in Figure 2. By integrated immunosensor, we first implemented quantitative detection for CA19-9, CEA and AFP and some detection performances such as sensitivity, specificity, and dynamic ranges are characterized. Conformed to the established calibration curves, the sensitivities for CA19-9, CEA and AFP are $10.0 \mathrm{U} / \mathrm{mL}, 1.0 \mathrm{ng} / \mathrm{mL}$ and $1.0 \mathrm{ng} / \mathrm{mL}$. The cross-reaction rates of each dot for antigen detection are no more than $5.2 \%$ evaluated by addition of heterogeneous antigen or related impurities. The dynamic ranges for CA19-9, CEA and AFP were 1.0-100.0 U/mL, 1.0-64.0 ng/mL and 1.0-128.0 ng/mL, respectively. Analytical reproducibilities were evaluated. The intra-assay CVs CA19-9, CEA and AFP determinations with the proposed method were between $1.6 \%$ and $8.9 \%$, the inter-assay CVs were between $2.1 \%$ and $7.8 \%$. Analytical recovery of the marker added to human serum ranged from $92.0 \%$ to $112.0 \%$ for all markers. The specificities tested for both cross-reaction and heterogeneous proteins of CA19-9, CEA and AFP were not more than $6.7 \%$.

\subsection{Methodology comparison with ECLIA}

Figure 4 shows a correlation of the IMBIE with conventional ECLIA. Correlation of test results by IMBIE with those by ECLIA for serum samples within the detection 
ranges of the standard curve gave the following regression equations: for CA19$9, \mathrm{Y}=0.57+1.03 \mathrm{x}(\mathrm{R}=0.96, \mathrm{n}=49)$; for $\mathrm{CEA}, \mathrm{Y}=0.518+0.92 \mathrm{x}(\mathrm{R}=0.98$, $\mathrm{n}=49)$; for AFP, $\mathrm{Y}=-1.14+1.005 \mathrm{x}(\mathrm{R}=0.99, \mathrm{n}=49)$. From a practical point of view, although ECLIA immunoassay sufficiently cover the dynamic range of the cancer markers to be measured for clinical evaluation, this kind of immunoassay take a rather long time ranged from 30min to several hours. The IMBIE, however, can cover the dynamic range within relative short assay time. Moreover, the proposed method has the advantages of simple operation by automated sampling and washing. Finally, even though the assay time is completed within $60 \mathrm{~min}$ regardless of the analyte, reasonable precision profiles and reliability of testing are obtained, making it possible to perform the IMBIE method with a fully automated and miniaturizing system.

\section{Conclusions}

Different strategies for AFP, CEA and CA 19-9 simultaneous immunoassay on the same substrate were developed. By unifying the reaction steps of CA19-9 for antigen direct detection, CEA for secondary antibody addition and AFP for secondary and enhancer application, considering the dynamic ranges and detection sensitivities, the multiplex analysis of these three markers were combined by IMBIE in serial format. The sensitivities and dynamic ranges for CA19-9, CEA and AFP were $10.0 \mathrm{U} / \mathrm{mL}, 1.0 \mathrm{ng} / \mathrm{mL}, 1.0 \mathrm{ng} / \mathrm{mL}$ and 2.0-100.0 U/mL, 0.1-64.0 ng/mL, 1.0$128.0 \mathrm{ng} / \mathrm{mL}$, respectively. The intra-assay and inter-assay CVs of CA19-9, CEA and AFP were between $1.6-8.9 \%$ and $2.1-7.8 \%$. The specificities tested for both crossreaction and heterogeneous proteins of CA19-9, CEA and AFP were not more than 6.7\%. Quantitative detection for 49 sera from gastric patient demonstrated that the accuracy for gastric cancer diagnosis was $83.4 \%$. The integrated analysis time is only $60 \mathrm{~min}$ required with an automated process. Therefore, the proposed method has the potential to apply in practice.

\section{Acknowledgments}

This work is supported by the cooperative project of academician workstation of Chongqing University of Science \& Technology (CKYS2014Y5), Research Foundation of Chongqing University of Science \& Technology (CK2014B4) and Chongqing pogram of Application Foundation and Advanced Technology(cstc2014jcyjA50032 cstc2014jcyjA10029).

\section{References}

1. X.Vrouwe, R.Luttge, and I.Vermes et al., Microchip Capillary Electrophoresis for Point-ofCare Analysis of Lithium. Clin.Chem. 53(1), 117-123(2007).

2. L.M.Wright, J. T. Kreikemeier, and C. J. Fimmel A concise review of serum markers for hepatocellular cancer. Cancer Detect and Prev. 31, 35-44 (2007).

3. J. R.Peng, H. S.Chen, and D. C.Mou et al., Expression of cancer/testis (CT) antigens in Chinese hepatocellular carcinoma and its correlation with clinical parameters. Cancer. Lett. 219,223-232 (2005). 
4. Ibtisam E. Tothill, Biosensors for cancer markers diagnosis. Seminars in Cell \& Developmental Biology 20, 55-62 (2009).

5. R. Z.Xu, X. X.Gan, and Y. M.Fang et al., A simple, rapid, and sensitive integrated protein microarray for simultaneous detection of multiple antigens and antibodies of five human hepatitis viruses (HBV, HCV, HDV, HEV, and HGV). Anal.Biochem. 362, 69-75 (2007).

6. G.Giannelli, E.Fransvea, and P.Trerotoli et al., Clinical validation of combined serological biomarkers for improved hepatocellular carcinoma diagnosis in 961 patients. Clin. Chim. Acta 383 (2007) 147-152

7. A. Washburn, M. Luchansky, and A. Bowman et al., Quantitative, Label-Free Detection of Five Protein Biomarkers Using Multiplexed Arrays of Silicon Photonic Microring Resonators. Anal.Chem. 82, 69-72(2010)

8. G.Jin, P.Tengvall, and I.Lundström et al., A Biosensor Concept Based on Imaging Ellipsometry for Visualization of Biomolecular Interactions. Anal.Biochem. 232,69-72(1995).

9. G. Jin, J. R., H. A. Imaging ellipsometry revisiteda developments for visualization of thin transparent layers on silicon substrates. Rev.Sci.Instrum. 67,2930-2936(1996).

10. Snopok, B. A., and I. V. Kruglenko Multisensor systems for chemical analysis: state-of-the-art in Electronic Nose technology and new trends in machine olfaction. Thin.Solid.Films 418(1): 21-41(2002).

11. W. B.Liang, W. J.Yi, and S. H.Li et al., A novel, label-free immunosensor for the detection of $\alpha$-fetoprotein using functionalised gold nanoparticles. Clin.Biochem. 42,1524-1530 (2009).

12. C. Huang, Y. Chen, and G. jin, A One-Step Immunoassay for Carbohydrate Antigen 19-9 by Biosensor Based on Imaging Ellipsometry. Annals of Biomedical Engineering, 39, 185192(2011).

13. C. Huang, Y. Chen, and C. Wang, et al., Detection of alpha-fetoprotein through biological signal amplification by biosensor based on imaging ellipsometry. Thin Solid Films, 519, 27632767(2011).

14. Z. H.Wang,Y. H.Meng, and P. Q.Ying et al., A label-free protein microfluidic array for parallel immunoassays. Electrophoresis.27,4078-4085(2006). 